\title{
Forward-Central Jet Correlations
}

\author{
Pedro Miguel RIBEIRO CIPRIANO, on behalf of CMS ${ }^{* \dagger}$ \\ $D E S Y$ - CMS \\ E-mail: pedro.ciprianoddesy.de
}

\begin{abstract}
The azimuthal correlation between forward and central jets has been measured in proton-proton collisions at the LHC, at the centre-of-mass energy of $7 \mathrm{TeV}$. The forward jet is reconstructed in the hadronic forward calorimeter, within the pseudorapidity $3.2<|\eta|<4.7$, while the central jet is limited to $|\eta|<2.8$. At least one central jet and one forward jet are required to have transverse momenta of $p_{T}>35 \mathrm{GeV}$. The measurement of the azimuthal angle between the jets is performed for different separations in pseudorapidity, with the largest separation being $\Delta \eta=7.5$ units. The analysis is carried out for inclusive dijet events and for two subsamples, one where an additional jet is required between the forward and the central jet, and one where the additional jet is vetoed. Comparisons between data and several different Monte Carlo models and tunes show a large sensitivity to the modeling of QCD radiation.
\end{abstract}

XXII. International Workshop on Deep-Inelastic Scattering and Related Subjects 28 April - 2 May 2014

Warsaw, Poland

* Speaker.

$\dagger$ A footnote may follow. 


\section{Introduction}

The dominant feature of strong (QCD) interactions in hadron-hadron collisions is the production of energetic partons in the final state, which manifest themselves - through the processes of quark and gluon radiation and hadronisation - as collimated bundles of hadrons, collectively called jets. In lowest order QCD (Born level), two jets are produced with equal transverse momenta, $p_{T}$, and back to back in the transverse plane of the event: the azimuthal angular separation $\Delta \phi_{\text {dijet }}=\left|\phi_{j e t 1}-\phi_{\text {jet } 2}\right|$ is equal to $\pi$. Additional radiation leads to decorrelations, i.e. to deviations of $\Delta \phi_{\text {dijet }}$ to smaller values. Soft radiation will create only small deviations in the correlation of the two highest- $p_{T}$ jets, while additional hard jets in the final state will lead to significant changes with respect to the dijet topology. Three-jet final states have been found to populate predominantly the region $2 \pi / 3<\Delta \phi_{\text {dijet }}<\pi$, while four-jet configurations lead to even smaller values than $2 \pi / 3$.

Dijet azimuthal correlations are therefore a valuable tool for studying QCD radiation and consequently also parton emission or "evolution" effects over a wide range of jet transverse momenta and jet multiplicities without the need to measure all the additional jets. However, multiple QCD radiation and multi-jet final states are still difficult to compute in perturbative QCD (pQCD), and the experiments rely heavily on Monte Carlo (MC) event generators for the preparation and the interpretation of the measurements. These MC generators usually use the DokshitzerGribov-Lipatov-Altarelli-Parisi (DGLAP) evolution equations [1-4] approximation. Alternative approaches like the Balitski-Fadin-Kuraev-Lipatov (BFKL) approximation [5-7] or the CiafaloniCatani-Fiorani-Marchesini (CCFM) approach [8-11] have since long been investigated, especially at HERA [12-17]. However, although in some cases significant discrepancies between data and DGLAP-based models have been observed, it has not been possible to pin down BFKL or CCFM effects in a convincing manner.

The LHC is particularly well suited for QCD studies of this type. With its high energy and the large pseudorapidity range available in its experiments, very long "parton ladders" open up along the coloured exchange and provide the opportunity for events with many emissions along this ladder. It is therefore assumed that QCD measurements of detailed final states with observables like the dijet azimuthal correlation at the LHC will shed new light on the principles of parton emission realised by nature.

Azimuthal correlations in the central region of the detector have been measured before by the Tevatron [18] and ATLAS [19] collaborations. At CMS, also dijet azimuthal correlations over large dijet rapidity separations [20] and for different $p_{T}$ regions of jet multiplicities [21] have been measured. In general, the measured distributions are found to be well described by commonlyused DGLAP-based event generators and also by next-to-leading order QCD calculations, with the exception of the region of small values of $\Delta \phi_{\text {dijet }}$ which is dominated by events with many jets in the final state.

This work focuses on a study of dijets which can have a large separation in pseudorapidity, $\Delta \eta=\left|\eta_{j e t 1}-\eta_{\text {jet } 2}\right|$. One jet is required to be in the central region of the detector and one in the forward region. The jet $p_{T}$ spectra of such events have already been measured at CMS [22]. The advantage of this experimental setup is that the sensitivity to the details of the parton radiation along the parton ladder is maximised and that, consequently, different parton evolution schemes might — finally — be experimentally confirmed or rejected. This is especially true for studies of the dijet 
decorrelation differentially in the pseudorapidity separation of the jets. These decorrelations allow to test the general properties of models in a more or less well understood phase space (small dijet separation) and then to look for effects of increasingly long parton ladders and increasingly many possible emissions - potentially revealing discrepancies between the different parton evolution approaches. Therefore, the measurement of the azimuthal correlation is done inclusively and also differentially in different pseudorapidity separations of the two jets constituting the dijet system.

More detailed studies of the hadronic final state than just dijet azimuthal correlations might shed additional light on the emission dynamics along the parton ladder. One example are studies of the additional jet activity in the events. Measuring a third hard emission in the dijet system would allow the influence of such radiation on the dijet correlation to be investigated. Therefore, in this measurement two sub-samples are derived from the inclusive measurement of dijet azimuthal correlations:

- Events with a third jet between the forward and central jets ("inside-jet tag").

- Events with a veto on a third jet between the forward and central jets ("inside-jet veto").

\section{Experimental Apparatus}

The CMS detector is described in Ref. [23]. The central part of the CMS detector contains a superconducting solenoid that provides an axial magnetic field of $3.8 \mathrm{~T}$ parallel to the beam axis. Charged particle trajectories are measured using silicon pixel and strip trackers that cover the pseudorapidity region $|\eta|<2.5$. An electromagnetic crystal calorimeter (ECAL) and a brass/scintillator hadron calorimeter (HCAL) surround the tracking volume and cover $|\eta|<3.0$. A forward quartzfibre Cherenkov hadron calorimeter (HF) extends the coverage to $|\eta|=5.2$.

\section{Event Selection}

The data samples used in the analysis were collected during 2010 in $p p$ collisions at a centre of mass energy $\sqrt{s}=7 \mathrm{TeV}$. A total integrated luminosity of $3.2 \mathrm{pb}^{-1}$ was used. Combined information from the calorimeters and trackers are used as input for the jets reconstruction [25] which is done using the anti- $k_{T}$ jet clustering algorithm [24], with a distance parameter of 0.5.

Events are selected containing at least one forward and one central jet with $p_{T}>35 \mathrm{GeV}$. The pseudorapidities $\eta$ of the central and the forward jet axis are required to be $|\eta|<2.8$ and $3.2<|\eta|<4.7$, respectively. In addition, the measurement is repeated for two subsamples: events with at least one additional jet with $p_{T}>20 \mathrm{GeV}$ between the forward and the central jet with respect to pseudorapidity, and events without any jet with $p_{T}>20 \mathrm{GeV}$ between the forward and the central jet. The two subsamples are referred to as "inside-jet tag" and "inside-jet veto". The additional jet is hereby referred to as the "inside jet".

\section{Systematic Uncertainties}

The systematic uncertainties are summarised in Table 1 . The total uncertainty, which is obtained by adding the individual uncertainties in quadrature, varies between 40 and $56 \%$ depending 
on the measured scenario and the observable. The different correlated systematic uncertainties are represented in the yellow band of the result. The uncorrelated uncertainties are shown as error bars connected to the data points.

Table 1: Systematic uncertainties of the measurement. The values represent the uncertainties for the observables averaged over the measured bins.

\begin{tabular}{|c|c|c|c|c|}
\hline Distribution & Statistics & MC Correction & JES & Total \\
\hline$\Delta \phi$ inclusive & $2 \%$ & $2 \%$ & $-38+48 \%$ & $-38+48 \%$ \\
in slices of $\Delta \eta$ & $4-5 \%$ & $2-4 \%$ & $-(39)+(49) \%$ & $-(39)+(49) \%$ \\
\hline$\Delta \phi$ inside-jet veto & $4 \%$ & $3 \%$ & $-39+48 \%$ & $-40+49 \%$ \\
in slices of $\Delta \eta$ & $5-12 \%$ & $3-15 \%$ & $-(40)+(50) \%$ & $-(44)+(55) \%$ \\
\hline$\Delta \phi$ inside-jet tag & $3 \%$ & $2 \%$ & $-38+51 \%$ & $-38+52 \%$ \\
in slices of $\Delta \eta$ & $6-7 \%$ & $2-4 \%$ & $-(39)+(52) \%$ & $-(40)+(52) \%$ \\
\hline
\end{tabular}

\section{Results}

The results of the inclusive measurement [26] are shown in Fig. 1. The cross section is presented as a function of the azimuthal difference between the forward and the central jet, $\Delta \phi$. The left plot compares data pared to PYтнia6 predictions with different tunes, as well as to a PYTHIA6 prediction performed without MPI. PYTHIA6 predicts events with jets which are somewhat more correlated than in the data, and no large difference between the various PYTHIA6 tunes is observed. The data is described by PYTHIA6 when considering the fairly large systematic uncertainties. Excluding the contribution from MPI results in a somewhat larger correlation between the forward and central jets, since the radiation from MPI mix with the hard scattering. In the right plot in the same figure, the data are compared to different MC generators. HERWIG++ provides the best shape and normalisation description, while PYTHIA8 and HERWIG6.5 describe the data whithin uncertainties, but Pythia8 predicts a slightly steeper $\Delta \phi$ distribution compared to the two HeRwIG predictions.
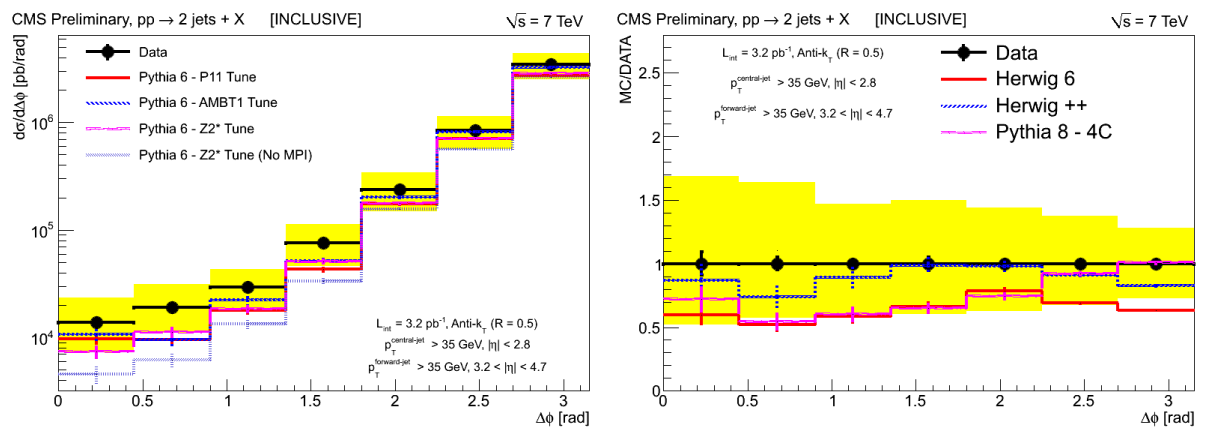

Figure 1: Cross section in bins of the difference in azimuthal angle between the forward and the central jet, $\Delta \phi$, and ratios between $\mathrm{MC}$ and data.

The cross-section differentially in bins of the pseudorapidity separation between the forward and central jets, $\Delta \eta$, is presented in Fig. 2. The $\Delta \phi$ distribution of the jets tends to flatten out 
with increasing $\Delta \eta$. Such behavior is expected from kinematics, a large separation between the jets allows for more QCD radiation between the jets, leading to a larger decorrelation between the forward and central jets. As for the $\Delta \eta$-integrated measurement, there is no striking difference between the tunes. The deviation between data and MC is about the same as in the inclusive measurement, and the data are described by РYтніA6 when considering the uncertainties of the data. HeRwIG++ describes the data very well, but the shape is slightly better reconstructed at low rapidity separations between the forward and the central jet. In general, there are no large differences between the inclusive and the double-differential measurement in terms of MC-data comparison.
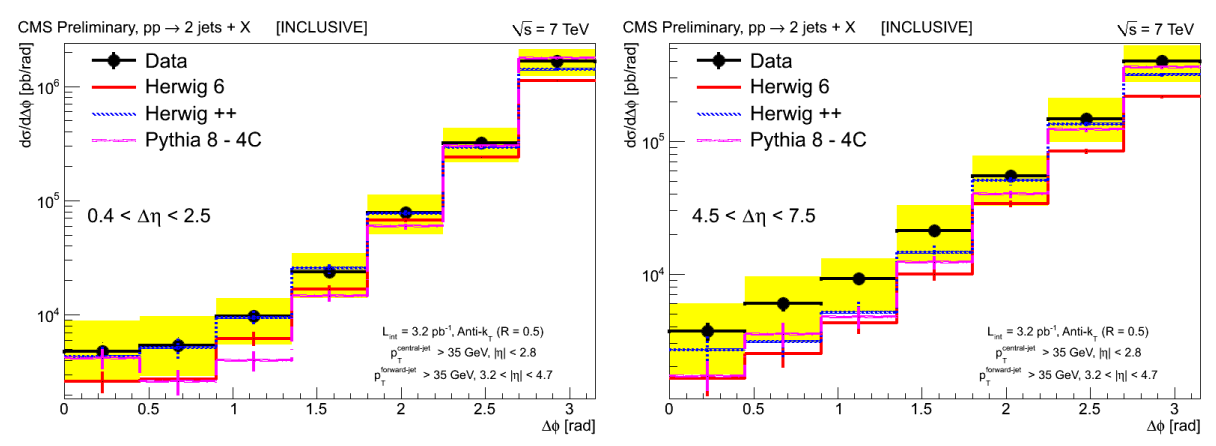

Figure 2: Double differential cross section for the inclusive scenario in bins of the difference in azimuthal angle between the forward and the central jet, $\Delta \phi$, in bins of the pseudorapidity separation between the two jets, $\Delta \eta$.

The measurement is repeated for the inside-jet tag scenario, in which a third jet is required between the forward and the central jet. The corresponding cross sections are shown in Figs. 3-4. As expected from kinematics the events with an inter-leading jet give flatter distributions, i.e. the jets are less correlated due to the fact that the additional jet removes momentum from the forwardcentral jet system. When comparing the data to Pythia6, Fig. 3, it is observed that the description of the shape by the MC is better than in the inclusive scenario. This is also the case for PYTHIA8, Herwig6.5 and Herwig++, as shown in Fig. 3 (left). In this scenario the effect from MPI in the correlations is smaller than in the inclusive scenario, although the total forward-central jet crosssection in Pythia6 decreases when the predictions are made without the MPI contribution. Again, the data are described by all the Monte Carlo predictions within the systematic uncertainty.

Figures 5-6 show the azimuthal jet correlations for the inside-jet veto scenario, in which events with a third jet between the forward and the central jet have been rejected. In contrast to the inside-jet tag scenario, the forward and central jets are now more correlated due to the suppressed radiation. The description of the data by the MC is in this case slightly worse than for the inclusive and inside-jet tag scenario. The general difference between the scenarios are further illustrated in Fig. 7 where the $\Delta \eta$ integrated measurements are compared in the same plot.

\section{Conclusions}

The tendency is that the correlation between the jets decreases with increasing $\Delta \eta$ and when 

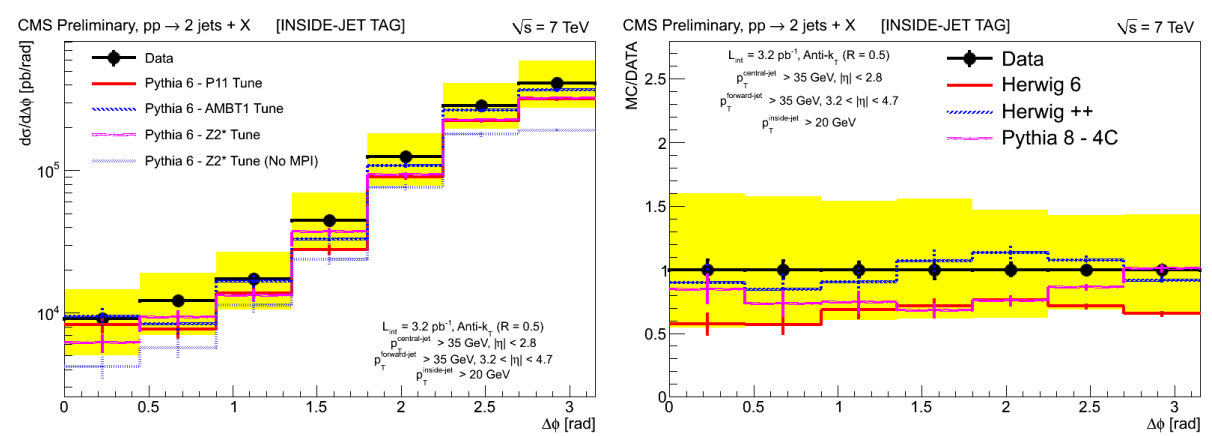

Figure 3: Cross section for the inside-jet tag scenario in bins of the difference in azimuthal angle between the forward and the central jet, $\Delta \phi$, and the ratio between $\mathrm{MC}$ and data.
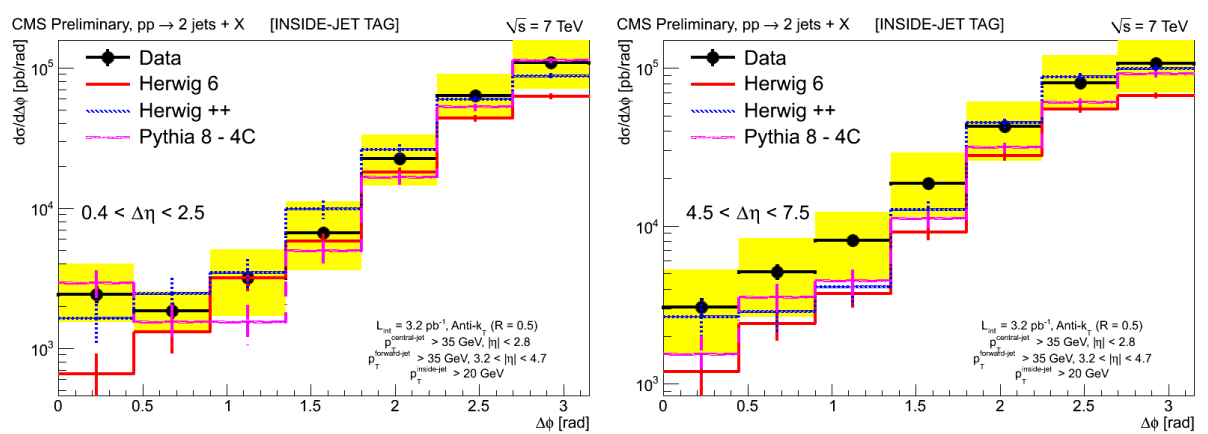

Figure 4: Double differential cross section for the inside-jet tag scenario in bins of the difference in azimuthal angle between the forward and the central jet, $\Delta \phi$, (top) in bins of the pseudorapidity separation between the two jets, $\Delta \eta$.
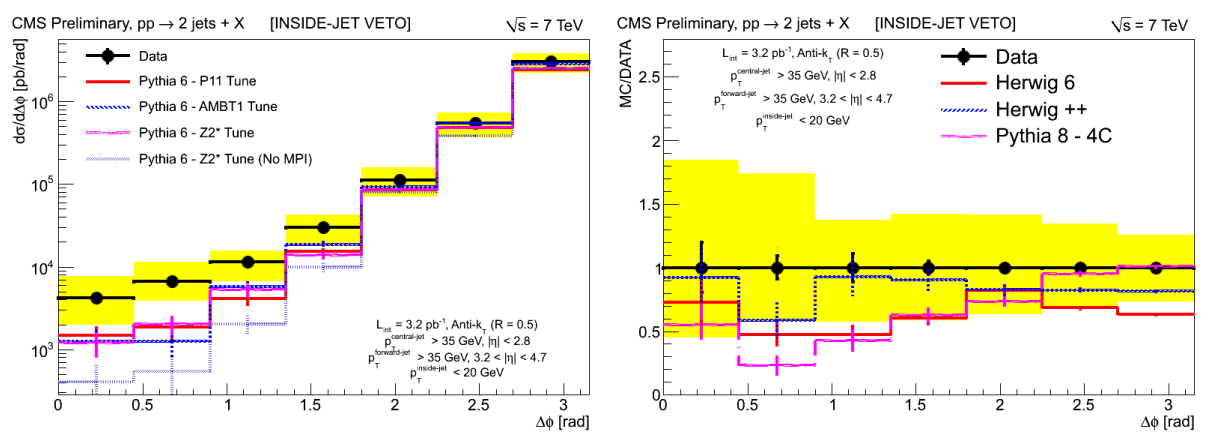

Figure 5: Cross section for the inside-jet veto scenario in bins of the difference in azimuthal angle between the forward and the central jet, $\Delta \phi$. 

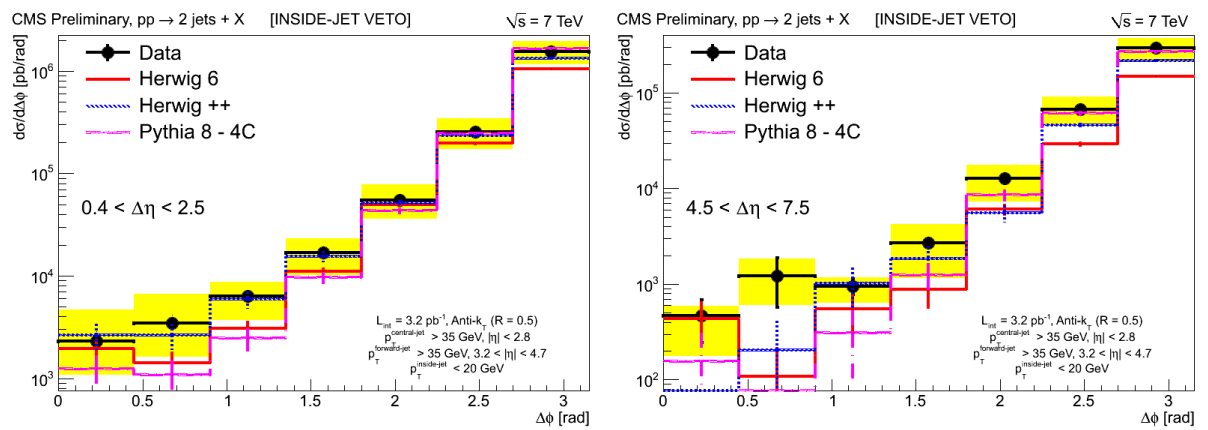

Figure 6: Double differential cross section for the inside-jet veto scenario in bins of the difference in azimuthal angle between the forward and the central jet, $\Delta \phi$, in bins of the pseudorapidity separation between the two jets, $\Delta \eta$.

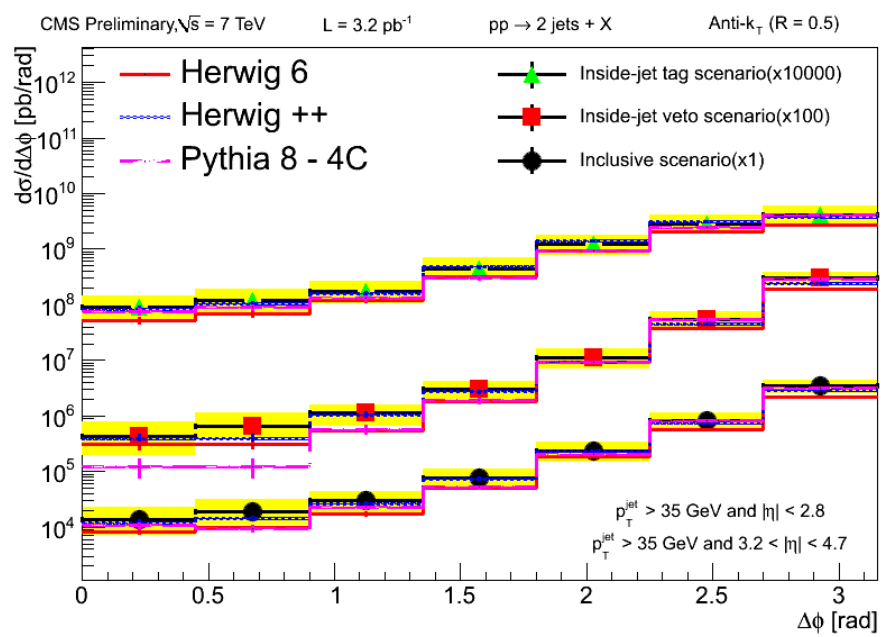

Figure 7: Comparison between the cross sections for the three different scenarios.

a third jet is required between the forward and the central jet. This effect is expected since the additional radiation in the event will naturally destroy the simple back-to-back topology of pure dijet events. Consequently, the correlation is strongest when the third-jet activity is vetoed.

The tested Monte Carlo generators (Herwig 6, Herwig++, Pythia 6 and Pythia 8) and respective tunes in general describe the measurement within the systematic uncertainties. HERWIG++ provides by far the best predictions describing both the shape and normalization of the data.

The different Pythia6 tunes do not differ much in their predictions. When the contribution from MPI is turned off the description of the dijet correlation is worse because the increased activity in the event no longer interfeers with the leading pair.

In summary, the DGLAP based generators describe well the complex observables of this analysis. No conclusion about evidence for BFKL like parton dynamics can be drawn from the presented measurement. 


\section{References}

[1] Gribov, V. N. and Lipatov, L. N., "Deep inelastic ep scattering in perturbation theory”, Sov. J. Nucl. Phys. 15, 438 (1972).

[2] Lipatov, L. N., “The parton model and perturbation theory”, Sov. J. Nucl. Phys. 20,94 (1975).

[3] Altarelli, Guido and Parisi, G., "Asymptotic Freedom in Parton Language”, Nucl. Phys. B 126, 298, doi:10.1016/0550-3213(77)90384-4 (1977).

[4] Dokshitzer, Yuri L., "Calculation of the Structure Functions for Deep Inelastic Scattering and $e+e$ Annihilation by Perturbation Theory in Quantum Chromodynamics", Sov. Phys. JETP 46, 641 (1977).

[5] Kuraev, E. A. and Lipatov, L. N. and Fadin, Victor S., "Multi - Reggeon Processes in the Yang-Mills Theory", Sov. Phys. JETP 44, 443 (1976).

[6] Kuraev, E. A. and Lipatov, L. N. and Fadin, Victor S., "The Pomeranchuk Singularity in Nonabelian Gauge Theories", Sov. Phys. JETP 45, 199 (1977).

[7] Balitsky, I. I. and Lipatov, L. N., “The Pomeranchuk Singularity in Quantum Chromodynamics”, Sov. J. Nucl. Phys. 28, 822 (1978).

[8] Ciafaloni, Marcello, “Coherence Effects in Initial Jets at Small $q^{2} / s$ ”, Nucl. Phys. B 296, 49, doi:10.1016/0550-3213(88)90380-X (1988).

[9] Catani, S. and Fiorani, F. and Marchesini, G., "QCD Coherence in Initial State Radiation”, Phys. Lett. B 234, 339. doi:10.1016/0370-2693(90)91938-8 (1990).

[10] Catani, S. and Fiorani, F. and Marchesini, G., "Small $x$ Behavior of Initial State Radiation in Perturbative QCD”, Nucl. Phys. B 336, 18, doi:10.1016/0550-3213(90)90342-B (1990).

[11] Marchesini, Giuseppe, "QCD coherence in the structure function and associated distributions at small x“, Nucl. Phys. B 445, 49, hep-ph/9412327 doi:10.1016/0550-3213(95)00149-M (1995).

[12] H1 Collaboration, "Forward $\pi^{0}$ Production and Associated Transverse Energy Flow in Deep-Inelastic Scattering at HERA”, Eur. Phys. J. C 36, 441, doi:10.1140/epjc/s2004-01868-3 (2004).

[13] , H1 Collaboration, "Forward Jet Production in Deep Inelastic Scattering at HERA", Eur. Phys. J. C" 46, 27 doi:10.1140/epjc/s2005-02471-x (2006).

[14] H1 Collaboration, "Measurement of the Azimuthal Correlation between the most Forward Jet and the Scattered Positron in Deep-Inelastic Scattering at HERA”, Eur. Phys. J. C 72, 1910 doi:10.1140/epjc/s10052-012-1910-8 (2012).

[15] ZEUS Collaboration, "Forward Jet Production in Deep Inelastic ep Scattering and low-x Parton Dynamics at HERA”, Phys. Let. B 632, 13 doi:10.1016/j.physletb.2005.09.066 (2006).

[16] ZEUS Collaboration, "Multijet production at low $x_{B j}$ in deep inelastic scattering at HERA", Nuc. Phys. B 46, 152, doi:10.1016/j.nuclphysb.2007.05.027 (2007).

[17] ZEUS Collaboration. "Forward-jet production in deep inelastic ep scattering at HERA", Eur. Phys. J. C 27, 515 doi:10.1140/epjc/s10052-007-0418-0 (2007).

[18] D0 Collaboration, "Measurement of dijet azimuthal decorrelations at central rapidities in $\mathrm{p}$ anti-p collisions at $s^{1 / 2}=1.96-T e V ”$, Phys. Rev. Lett. 94, 221801, hep-ex/0409040, doi:10.1103/PhysRevLett.94.221801 (2005).

[19] ATLAS Collaboration, "Measurement of Dijet Azimuthal Decorrelations in pp Collisions at $\sqrt{s}=7$ TeV”, Phys. Rev. Lett. 106, 172002, arXiv:1102.2696, doi:10.1103/PhysRevLett.106.172002 (2011). 
[20] CMS Collaboration, "Dijet Azimuthal Decorrelations in $p p$ Collisions at $\sqrt{s}=7$ TeV”, Phys. Rev. Lett. 106, 122003, arXiv:1101.5029, doi:10.1103/PhysRevLett.106.122003 (2011).

[21] CMS Collaboration, "Ratios of dijet production cross sections as a function of the absolute difference in rapidity between jets in proton-proton collisions at $\sqrt{s}=7 \mathrm{TeV}$ ", Eur. Phys. J. C 72, 2216, arXiv:1204.0696, doi:10.1140/epjc/s10052-012-2216-6 (2012).

[22] CMS Collaboration, "Measurement of the inclusive production cross sections for forward jets and for dijet events with one forward and one central jet in pp collisions at $\sqrt{s}=7 \mathrm{TeV}$ ", JHEP 1206, 36, arXiv:1202.0704, doi:10.1007/JHEP06(2012)036 (2012).

[23] CMS Collaboration, “The CMS experiment at the CERN LHC”, JINST 0803, S08004, doi:10.1088/1748-0221/3/08/S08004 (2008).

[24] Cacciari, M. and Salam, G. P. and Soyez, G., “The anti- $k_{t}$ jet clustering algorithm”, JHEP 0804, 063, arXiv:0802.1189, doi:10.1088/1126-6708/2008/04/063 (2008).

[25] CMS Collaboration, "Particle-Flow Event Reconstruction in CMS and Performance for Jets, Taus, and $E_{T}^{m i s s}$ ", CMS Physics Analysis Summary, CMS PAS PFT-09-001 (2009)

[26] CMS Collaboration, "Measurement of azimuthal correlations between forward and central jets in proton proton collisions at sqrt(s)=7 TeV", CMS Physics Analysis Summary, CMS-PAS-FSQ-12-008 (2014) 